\title{
ACCIONES PARA INDUCIR LAS COMPRAS EN EL COMPORTAMIENTO DE LOS CONSUMIDORES DE LA TIENDA GIORGIO G.
}

ACTIONS TO INDUCE SHOPPING IN THE CONSUMERS BEHAVIOR OF THE GIORGIO G SHOP

Dra. Adriana del Villar Ayala ${ }^{a}$

Mtro. José Carlos Vélez González ${ }^{b}$

Mtra. Sonia Edith Villeda Martínez ${ }^{c}$

\author{
a Benemérita Universidad Autónoma de Puebla \\ Facultad de Contaduría Pública, adelvillara@hotmail.com \\ b Benemérita Universidad Autónoma de Puebla \\ Facultad de Contaduría Pública, vegc1129@yahoo.com.mx \\ c Benemérita Universidad Autónoma de Puebla \\ Facultad de Contaduría Pública, vi.ms.25@hotmail.com
}

\section{RESUMEN}

En tiempos actuales el estudio del comportamiento del consumidor en tiendas constituye un factor clave para alcanzar el éxito a partir de la utilización acertada de las técnicas de merchandising que realmente incidan en los consumidores y en consecuencia permitan elevar las ventas, garantizando así la rentabilidad del negocio. El presente trabajo fue realizado con el objetivo de diseñar un plan de acciones para elevar las ventas de la tienda de productos de vestir exclusivas Giorgio G. Para ello se utilizaron métodos teóricos: análisis-síntesis, histórico-lógico e inductivo-deductivo y métodos empíricos: análisis documental, las encuestas y entrevistas, siguiendo la lógica de la Metodología General para la Solución de Problemas propuesta por Leyva del Toro (2018), se diagnosticaron las principales problemáticas que están incidiendo en que no se alcancen los niveles de ventas deseados, el análisis, sus causas y en correspondencia la propuesta de un plan de acciones que permita aplicar las técnicas de merchandising más proclives a incidir en sus consumidores.

PALABRAS CLAVE: Comportamiento; Consumidor; Técnicas de merchandising. 


\section{ABSTRACT}

The study of the consumer behavior at stores constitutes a factor in modern times key to achieve the success as from the correct employment of Merchandising's techniques that really, they affect on the consumers and in consequence allow to raise sales, guaranteeing the profitability of business that way. The present work was executed with the objective to design a plan of actions to raise the sales of the store of dressing products, exclusive Giorgio G. Were used several theoretic methods: Analytical synthesis, historic logician and inductive deductive and empiric methods: Documentary analysis, the opinion polls and interviews, following the logic of the General Methodology for the Solution of Problems proposed by Leyva del Toro (2018). The main problems that are affecting diagnosed themselves in than not catch up with him the levels of desired sales, the analysis his causes and in the mail the proposal of a plan of actions that it enable applying more Merchandising's techniques prone to have an effect on his consumers.

KEY WORDS: Behavior; Consumer; Merchandising techniques.

\section{INTRODUCCIÓN}

El mundo contemporáneo impone a la esfera de los servicios entre otros retos el de lograr satisfacer las necesidades y expectativas de los clientes a partir de la personalización y conocimiento profundo de sus mercados. Las cadenas de tiendas hoteleras y extra hoteleras no son la excepción, pues las mismas están diseñadas para complacer las necesidades de sus segmentos de clientes a partir de un servicio distinguido por la excelencia. Sin embargo, para lograr su supervivencia en el mercado han de aplicar diversas técnicas que tengan en cuenta las nuevas tendencias de la industria que son necesariamente un punto de partida para alcanzar el éxito (Serrano, Escalona \& Pompa, 2018).

En este ámbito juega un papel primordial el conocimiento del comportamiento del consumidor como tip para personalizar el servicio, pues los cambios ocurridos en el sector han provocado una explosión en las elecciones de los consumidores, desde comprar en tiendas tradicionales y familiares (conocidas como kirana), hasta supermercados y centros comerciales modernos, así como en tiendas electrónicas en línea. En este sentido destacan las características de los formatos de compra tradicionales y modernos que influyen en el comportamiento del consumidor, así como la naturaleza y el nivel de las prácticas competitivas de marketing (Dholakia, Dholakia, \& Chattopadhyay, 2018).

Teniendo en cuenta lo anterior los minoristas tienen cada vez más acceso a los datos de seguimiento de las acciones de un consumidor como un flujo de eventos a lo largo del tiempo, lo que puede ser un habilitador clave del análisis de marketing (Wedel\&Kannan, 
2016) citado por (Kakatkar \& Spann, 2018), pues en el contexto de venta al por menor, los consumidores pueden tomar señales del ambiente de la tienda para hacer juicios e inferencias sobre la misma, lo cual ha llevado a que los gerentes minoristas se centren en cómo los elementos ambientales de un espacio minorista están diseñados y dispuestos para influir en el comportamiento del consumo (Baker, Parasuraman, Grewal, \&Voss, 2002; Bitner, 1992) citado por (Baek, Choo, \& Lee, 2018).

Precisamente una de las problemáticas presentadas por la tienda Giorgio G, especializada en productos de vestir exclusivos, ubicada en el destino turístico Holguín es el incumplimiento de los planes de ventas e insatisfacción de los clientes con sus servicios, revelando que existen insuficiencias tales como:

- Poco dominio de las técnicas de merchandising para la exhibición de los productos.

- Insuficiente dominio de las características del surtido, tipología de comercio y segmento de mercado que sirve, para realizar la gestión de ventas.

- Insatisfacción de los clientes con el surtido ofrecido por la sucursal en las diferentes unidades.

Teniendo en cuenta estas problemáticas se define como objetivo general diseñar un plan de acciones para elevar las ventas de la tienda Giorgio $\mathrm{G}$.

\section{EL COMPORTAMIENTO DEL CONSUMIDOR EN TIENDAS. DEFINICIONES}

El comportamiento del consumidor es el estudio del comportamiento que los consumidores muestran al buscar, comprar, utilizar, evaluar y desechar los productos y servicios que, consideran, satisfarán sus necesidades. El comportamiento del consumidor, como una disciplina del Marketing existe desde los años 60 y se enfoca en la forma que los individuos toman decisiones para gastar sus recursos disponibles (tiempo, dinero y esfuerzo) en artículos relacionados con el consumo (Schiffman, 2010).

Según (Richers, 1984) el comportamiento del consumidor se caracteriza por las actividades mentales y emocionales realizadas al seleccionar, comprar y usar los productos o servicios para satisfacer sus necesidades y deseos, lo cual coincide con lo planteado por (Churchill \& Peter, 2000; Rodrigues \& Delane, 2012) al decir que el comportamiento de los consumidores está formado por pensamientos, sentimientos y acciones, que influyen sobre ellos y provocan cambios.

Por otra parte (Perelló, 2005) plantea que el comportamiento del consumidor constituye aquella parte del comportamiento humano relacionada con la adquisición, uso y disfrute de bienes y recursos tangibles e intangibles de carácter económico, así como con el proceso de decisión que conduce a estos actos. 
Teniendo en cuenta lo anterior, el presente informe se adscribe a la definición planteada por (Moutinho, 2000), al expresar que el comportamiento del consumidor se refiere al proceso de adquirir y organizar la información, orientado a una decisión de compra y de la utilización y evaluación de los productos y servicios. Este proceso conlleva las etapas de búsqueda, compra, utilización y evaluación (Cassanego, Maria \& Caren, 2011). Se incluye en su definición el proceso de decisión de compra, elemento esencial para la fundamentación de la presente investigación, además de otros aspectos que se consideran de gran importancia.

Lo anterior se justifica con lo planteado por (Kotler \& Kevin, 2006) al expresar que para comprender cómo se toman en realidad las decisiones de compra, los mercadólogos deben detectar quién toma la decisión de compra y quién influye en la misma. Las personas que participan pueden ser iniciadores, influyentes, encargados de tomar las decisiones, compradores o usuarios.

En congruencia con lo anterior, el estudio del comportamiento del consumidor consiste en analizar cómo los individuos, los grupos y las empresas seleccionan, adquieren, utilizan y disponen de bienes, servicios, ideas o experiencias para satisfacer sus necesidades y deseos. Este análisis ofrece guías para mejorar o lanzar productos o servicios, fijar precios, seleccionar canales, elaborar mensajes y desarrollar otras actividades de marketing. Las empresas siguen de cerca las nuevas tendencias del mercado en busca de nuevas oportunidades de marketing (Kotler \& Kevin, 2006).

\section{CARACTERIZACIÓN DE LA TIENDA GIORGIO G. PERTENECIENTE A LA SUCURSAL CARACOL HOLGUÍN}

La tienda Giorgio G. perteneciente a la Sucursal Caracol Holguín se exhibe al cliente como una tienda individual, pues está se presenta en un solo local de forma individual. Su estrategia de venta se basa en el autoservicio, el comprador selecciona sus productos de la estantería y los paga en las cajas a la salida. Según la ubicación es intrahotelera y teniendo en cuenta los clientes que atienden es no turística. Finalmente, según la especialización se clasifica en negociada, pues según contrato, un proveedor garantiza la mercancía etc. En este caso los productos que oferta son de la marca Giorgio, de gran prestigio a nivel internacional.

La cartera de productos está compuesta de la siguiente forma:

Genéricos y subgenéricos

- Confecciones

- Confecciones de vestir.

- Confecciones casuales.

- Ropa interior. 
- Confecciones playeras.

- Maletas Carteras y Accesorios.

- Calzado.

La tienda fue remodelada en el pasado año, para relanzar un producto rejuvenecido de gran confort. Según los resultados económicos de los meses posteriores, diciembre y enero estos se comportaron de la siguiente forma.

\section{Diciembre}

La entidad debe ingresar de forma mensual 19,549, sin embargo, en el mes de diciembre de 2018 se cumplió al 49\% respecto a dicho nivel deseado con un total de ingresos de 9,541.40 (Figura 1). En la estructura de ingresos la tienda Giorgio representa el $1.4 \%$ del total de tiendas.

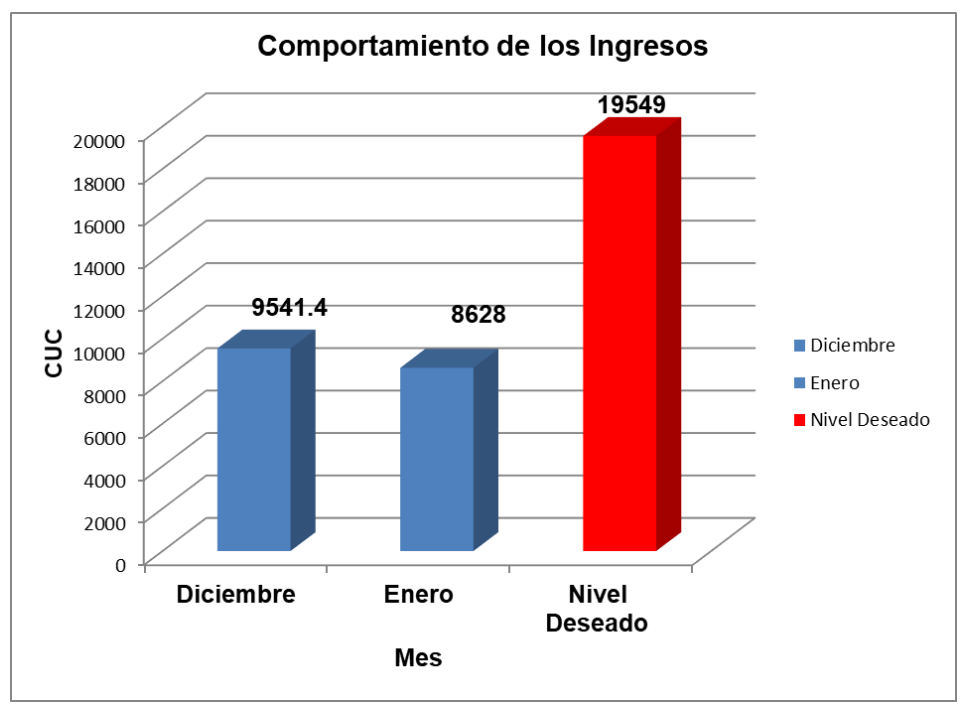

Figura1. Comportamiento de los ingresos. Fuente: Elaboración propia.

El costo de venta se sobrejecuta en un $24.8 \%$, lo que representa un incremento de $1,209.86$ con relación al plan (Figura 2). El costo por peso de venta planificado es de 0.519 y el real obtenido es de 0.638 , lo que evidencia un aumento de 0.119 con respecto a los niveles planificados. 


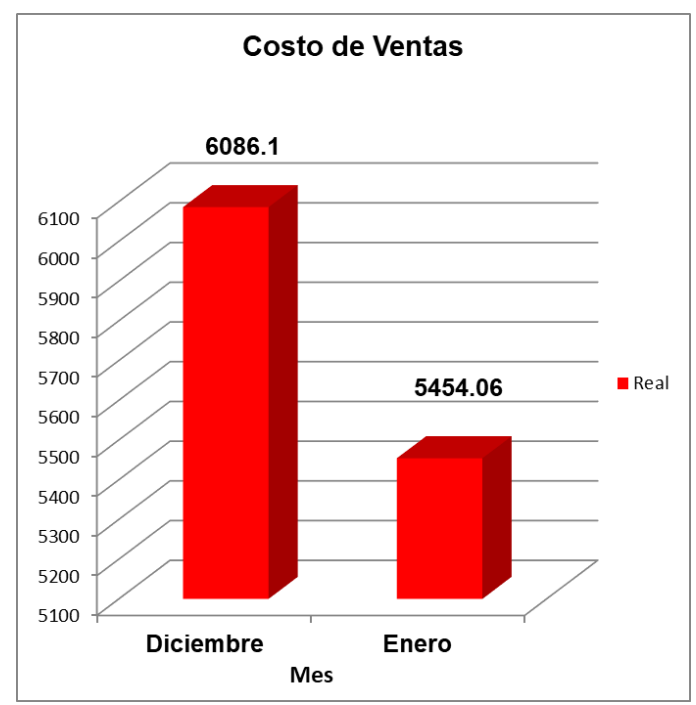

Figura 2. Comportamiento del costo de venta. Fuente: Elaboración propia.

\section{Enero}

En el mes enero de 2018 se ingresaron 8,628 lo que representa un cumplimiento del 44\% respecto al nivel deseado de ingresos mensual, ya mencionado con anterioridad. En la estructura de ingresos la tienda Giorgio representa el 1.3\% del total de tiendas (Figura 3)

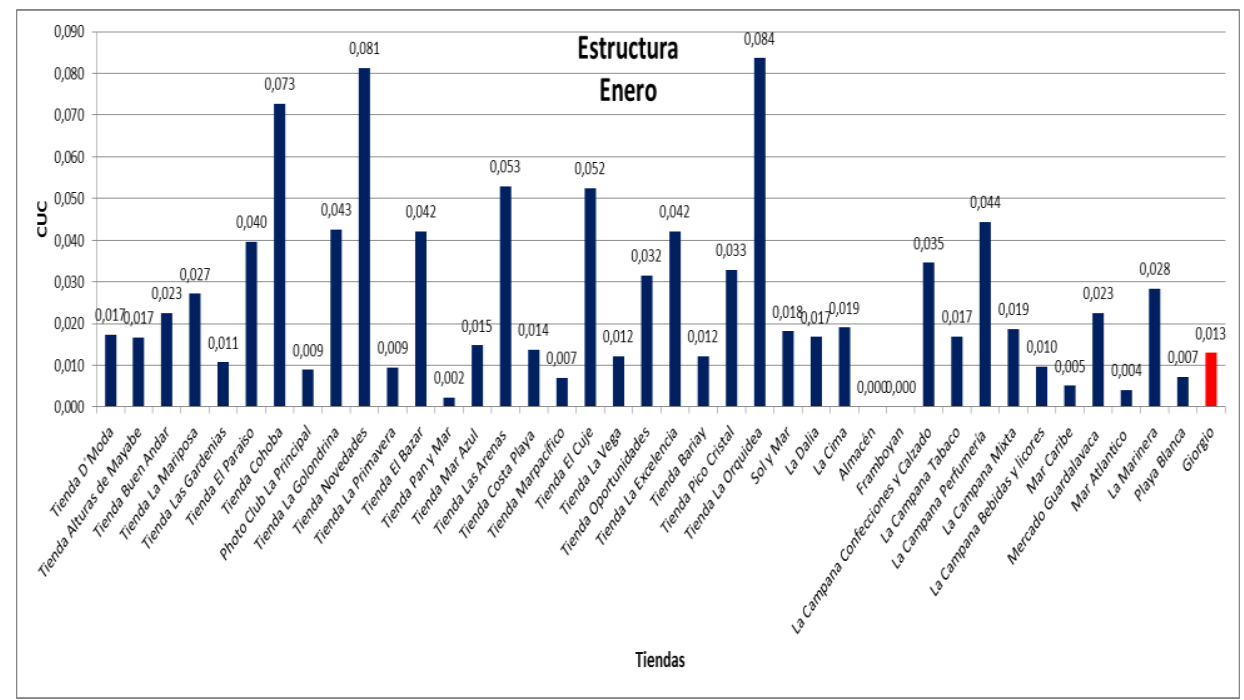

Figura 3. Posición de la tienda dentro de la estructura de la Sucursal

El costo de venta se sobre ejecuta en un $4.8 \%$, lo que representa un incremento de 249.6 con relación al plan (Figura 2). El costo por peso de venta planificado es de $0.670 \mathrm{CUC}$ y el 
real obtenido es de 0.632 , lo que evidencia un aumento de 0.038 con respecto a los niveles planificados.

Siguiendo la lógica de la Metodología General para la Solución de Problemas propuesta por Leyva del Toro (2018) para tiendas extra hoteleras, se detectó como problema central el bajo nivel de ventas en un 49\% y 44\% en el mes de diciembre de 2018 y enero del 2019 respectivamente en la Tienda Giorgio G. En consecuencia, se declara como situación deseada alcanzar un $100 \%$ de las ventas esperadas de la tienda Giorgio G. en el próximo período.

\section{DIAGNÓSTICO DE LAS PRÁCTICAS DE MERCHANDISING UTILIZADAS EN LA TIENDA GIORGIO G.}

En primer lugar, la arquitectura del sitio, en este caso principalmente la arquitectura exterior se muestra moderna y sugerente, el rótulo con el nombre de la instalación es llamativo y perfectamente compatible con la imagen que presenta el establecimiento, además la limpieza de los cristales facilita la visualización a la arquitectura interior que se proyecta confortable. Predominan los colores negro, gris y blanco, muy tendenciales y que indican sensación de espacio, a pesar de las pequeñas dimensiones de la infraestructura.

Se percibe un olor neutro y no se dispone de música en el mismo. En el interior se exponen además varios maniquíes con sugerencias de combinaciones en cuanto a vestuario y accesorios, que se cambian frecuentemente.

Los productos se exhiben en percheros, mesas y estantes. Estos últimos llegan a alcanzar una altura superior a $1.70 \mathrm{~m}$, por lo que reduce la posibilidad de venta de estos artículos a un $10 \%$.

Por otra parte, desde su reapertura solo se ha realizado un solo modelaje, que tuvo lugar en las cercanías de la piscina del Pernik, hotel donde se encuentra enclavada. Mientras que el día 26 de febrero se hicieron rebajas de productos.

\section{DISEÑO DE UN INSTRUMENTO QUE PERMITA CARACTERIZAR EL COMPORTAMIENTO DE LOS CONSUMIDORES DE LA TIENDA GIORGIO G.}

A partir de la información contenida en el marco teórico referencial sobre comportamiento del consumidor en tiendas se elaboró el siguiente mapa mental que resume el flujo del comportamiento del consumidor que efectúa la compra en tiendas vinculado a aspectos del Merchandising que pueden incidir en que el cliente elija determinado producto (Figura 4). 


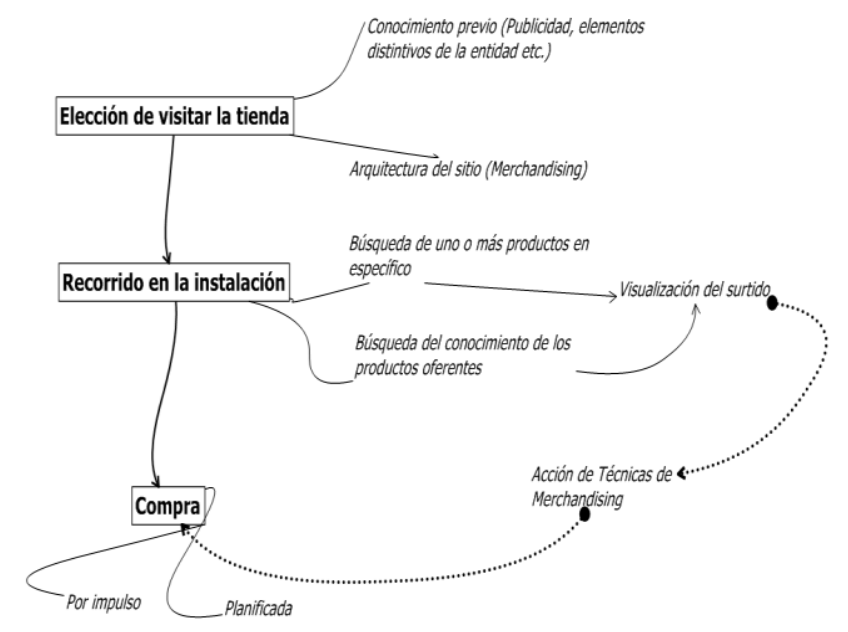

Figura 4. Flujograma Comportamiento de consumidor que efectúa compras en tiendas. Fuente: Elaboración propia.

Teniendo en cuenta los factores principales que inciden en el comportamiento del consumidor en las tiendas y que se reflejan en el mapa mental elaborado se seleccionó el instrumento propuesto por Baek, Choo \& Lee (2018) para evaluar el comportamiento del consumidor en la tienda Georgio G.

Estimado cliente:

Esta investigación tiene como objetivo identificar los principales elementos que influyen en que usted pueda realizar la compra de nuestros productos. Los resultados de la investigación tendrán fines únicamente académicos y garantizamos que será totalmente anónima.

Edad

Sexo

1. Usted decidió entrar a la tienda porque:

Tenía decidido visitarla

Un producto llamó mi atención

La arquitectura del sitio me impulsó a hacerlo

Otro, ¿̇cuál?

2. ¿Busca usted algún producto en específico?

Sí _ No

3. En caso de haberlo buscado, ¿̇lo encontró?

Del Villar, A., Vélez, J.C. \& Villeda, S.E.

Acciones para inducir las compras en el comportamiento de los consumidores de la tienda GIORGIO G 
Sí No

4. Cuando usted no localiza un producto que busca en una tienda, ¿qué hace?

pregunto al dependiente

compro otro

voy a otra tienda en busca de este

desisto de la compra

otra idea, ¿̇uál?

5. Compró usted algún producto que no tenía pensado

Sí No

6. En su recorrido por la instalación ċle llamó la atención algún producto que no buscaba?

Sí No

7. ¿Ha comprado en nuestra tienda en otras ocasiones?

Sí _No

8. ¿Siempre que necesita un producto que nuestra tienda oferta nos visita?

Sí _ No

9. ¿Conoce usted acerca de la marca de los productos que comercializamos?

Sí No

10. Si tuviera que valorar la relación calidad-precio de los productos que ofertamos en una escala del 1 al 4, ¿qué puntuación nos daría?

1 2 3 4

Muy buena

Muy mala

\section{DESCRIPCIÓN DE LA MUESTRA PARA LA APLICACIÓN DEL INSTRUMENTO}

La población objeto de estudio estuvo conformada por los clientes que visitaron la instalación en fecha posterior al día 12 de febrero de 2019 hasta el 23 de febrero de 2019, la misma fué tomada en base a la cantidad promedio de clientes que diariamente visitan la instalación. Se utilizó la fórmula para calcular el tamaño de muestra cuando se conoce la población, donde:

$$
\mathrm{n}=\frac{\mathrm{k}^{2} \mathrm{pqN}}{\mathrm{e}^{2}(\mathrm{~N}-1)+\mathrm{K}^{2} \mathrm{pq}}=\frac{1,96^{2} * 0,5 * 0,5 * 200}{(0,1)^{2}(199)+1,96^{2} 0,5 * 0,5}=65
$$

$\mathrm{n}$ : tamaño de muestra 
N: población

$\mathrm{k}=$ constante

p: probabilidad con la que se presenta el fenómeno

q: (1-p) probabilidad de que no se presente el fenómeno

e: error en tanto por ciento. Para este tipo de investigación se recomienda el error de $10 \%$.

Para una muestra de 385 unidades de análisis. Los sujetos se seleccionaron de forma aleatoria.

\section{PROCESAMIENTO Y ANÁLISIS DE LOS RESULTADOS ARROJADOS POR EL INSTRUMENTO APLICADO.}

Una vez aplicada las encuestas los resultados fueron computarizados con ayuda de Microsoft Excel, para ello se utilizó el libro de código, obteniendo una matriz que permitió su análisis en el software SPSS 21. Como resultado del procesamiento en dicho software se pudo comprobar que el instrumento posee una fiabilidad muy alta al obtener un Alfa de Cronbach 0.80 y además válido pues el índice KMO fue de 0.76. La tabla 1 muestra la salida de datos del SPSS 21.

\section{Tabla 1.}

Salidas de datos SPSS 21

\begin{tabular}{|c|c|}
\hline \multicolumn{2}{|c|}{ Estadísticos de fiabilidad } \\
\hline $\begin{array}{c}\text { Alfa de } \\
\text { Cronbach }\end{array}$ & $\begin{array}{c}\text { N de } \\
\text { elementos }\end{array}$ \\
\hline, 806 & 10 \\
\hline
\end{tabular}

\section{RESULTADOS Y DISCUSIÓN}

Ítem 1. Motivo de la visita

De los 65 encuestados solo 15 tenían decidido visitar la tienda, 25 lo hicieron porque la arquitectura del sitio les impulsó a hacerlo y 25 porque un producto les llamó la atención. Los resultados en este ítem son bastante favorables pues aproximadamente el $77 \%$ estuvo inducido por aspectos que incluye el Merchandising, en este caso: arquitectura del sitio y exposición de productos sugerentes (Figura 5). 


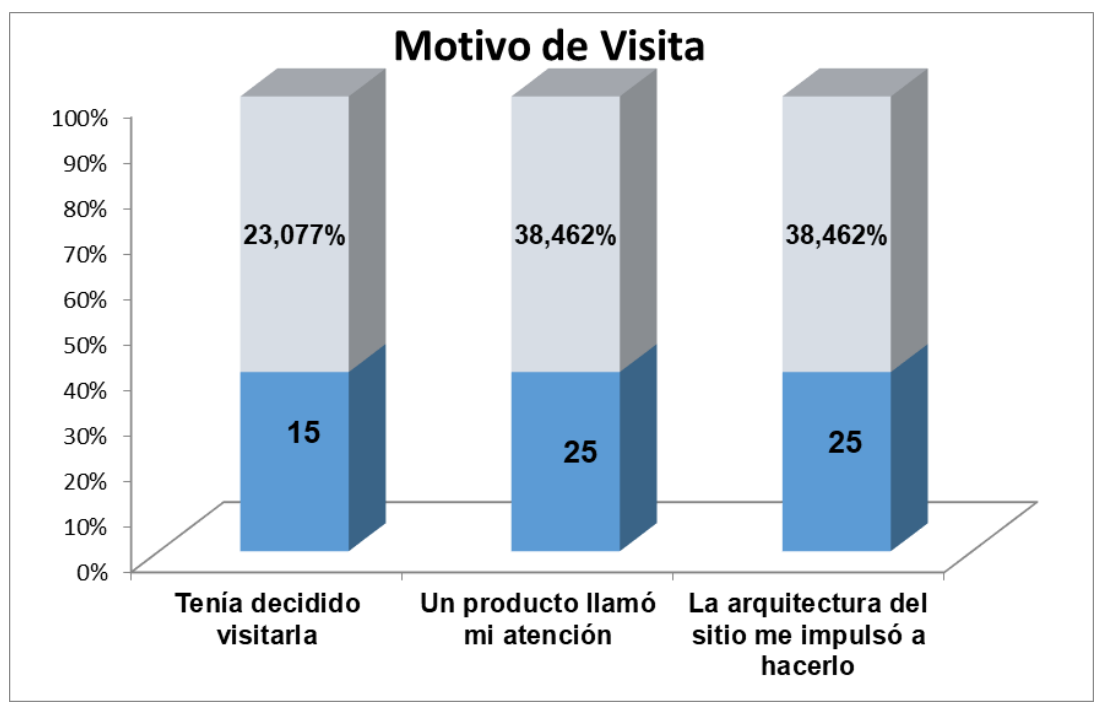

Figura 5. Motivo de visita. Fuente: Elaboración propia.

Ítem 2. Búsqueda del producto e ítem 3. Accesibilidad del producto

Del total de encuestados 41 buscaban un producto exclusivo de la tienda, lo cual representa el $63 \%$ del total (Figura 6). De esos 41 solo 21 encontraron el producto que buscaban, por lo que aproximadamente un $30 \%$ no lo encontró. (Figura 7)

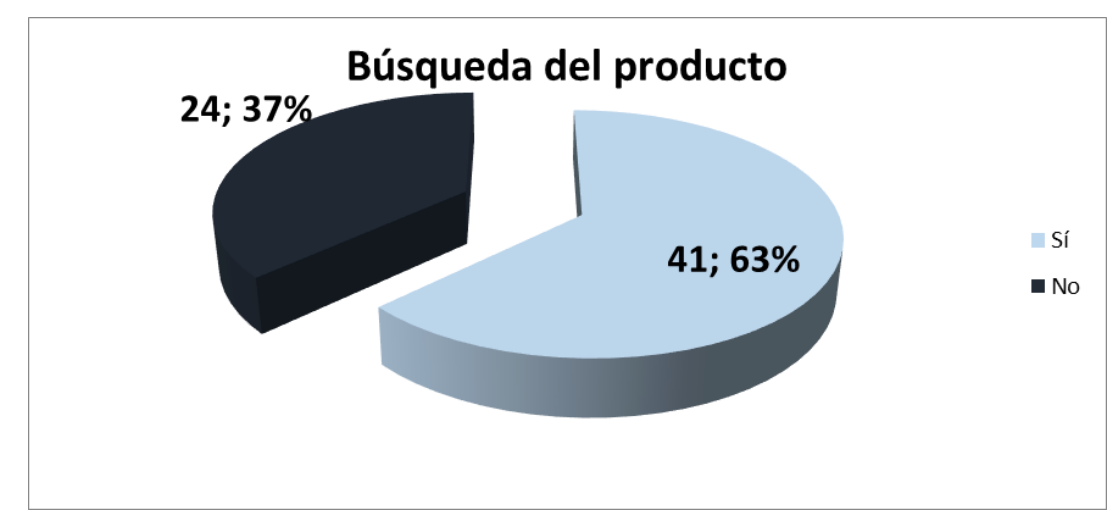

Figura 6. Búsqueda del producto. Fuente: Elaboración propia. 


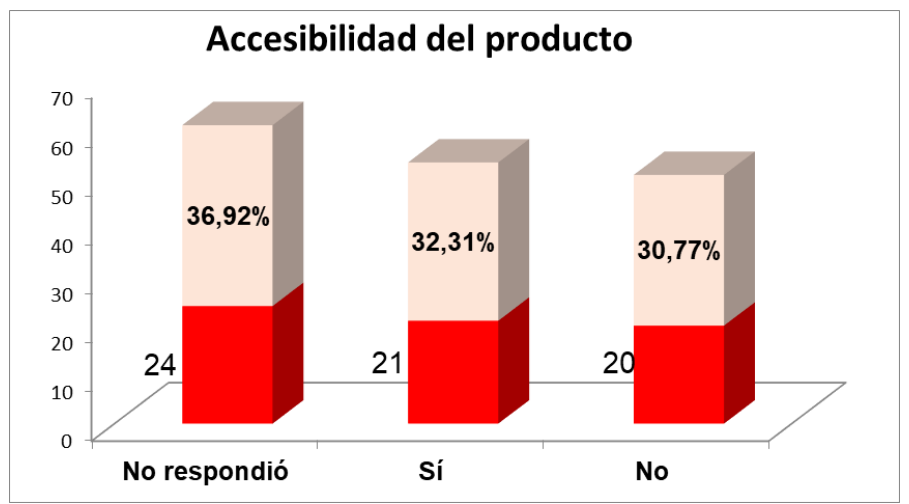

Figura 7. Accesibilidad del producto. Fuente: Elaboración propia.

\section{Ítem 4. Reacción a la inaccesibilidad}

Los resultados de la encuesta en cuanto a la interrogante de: qué hace cuando usted no localiza un producto que busca en una tienda, evidencian que la inaccesibilidad del producto pudo causar la pérdida de un $50 \%$ de las ventas, pues un $40 \%$ afirma dirigirse a otro establecimiento. Además, demostró que a pesar de ser una tienda diseñada para el autoservicio la gestión de la fuerza de venta resulta esencial, pues el $43.08 \%$ de los clientes busca el asesoramiento de los dependientes (Figura 8).

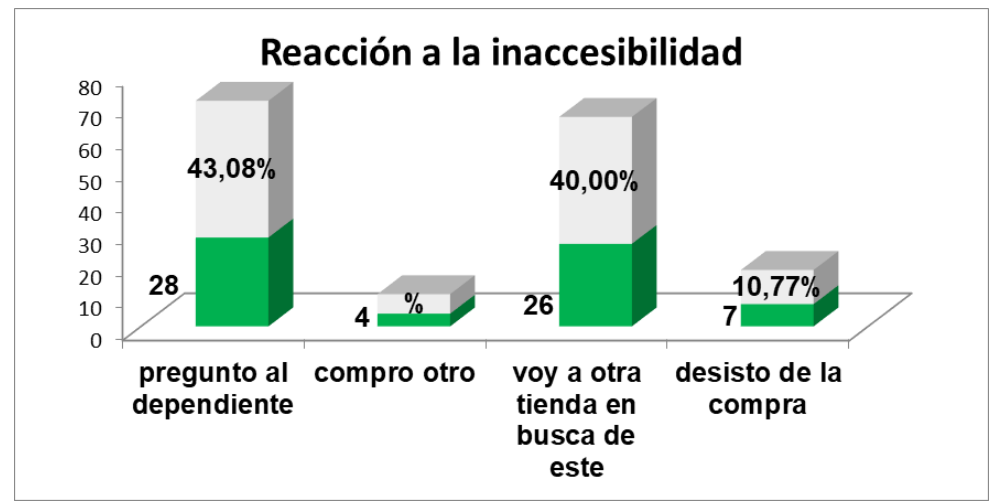

Figura 8. Reacción a la inaccesibilidad. Fuente: Elaboración propia.

Ítem 5. Ventas por Impulso

Del total de clientes encuestados solo 10 compraron algún producto que no tenían pensado adquirir, por lo que las ventas por impulso del total de unidades de análisis estuvieron representadas por solo el 10\%, para un nivel bajo de este indicador (Figura 9). 


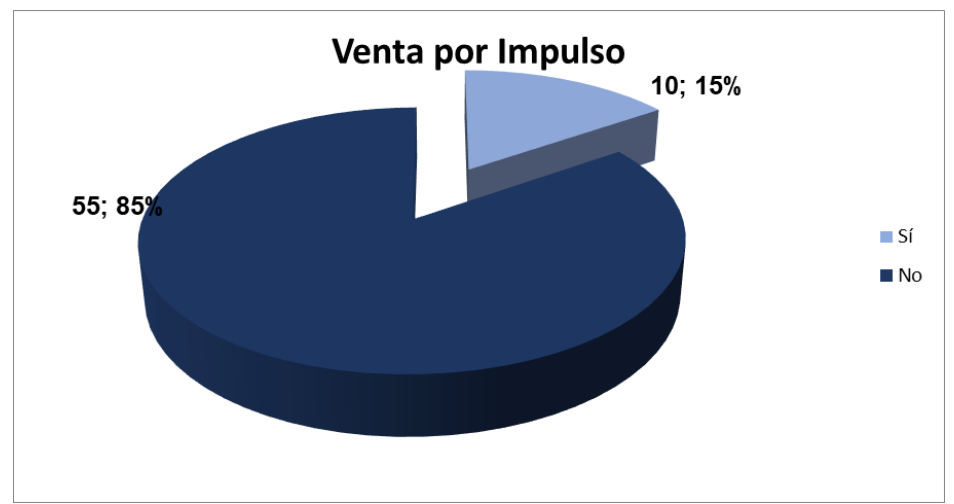

Figura 9. Venta por Impulso. Fuente: Elaboración propia.

\section{Ítem 6. Producto sugerente}

Del total de encuestados solo a un $22 \%$ les llamó la atención alguno de los productos que se exhibían, aspecto importante, pues una compra por impulso puede iniciarse por este indicador, se han de aplicar las técnicas de Merchandising necesarias para resaltar las características de algún producto en particular que se busque vender según la estrategia del establecimiento (Figura 10).

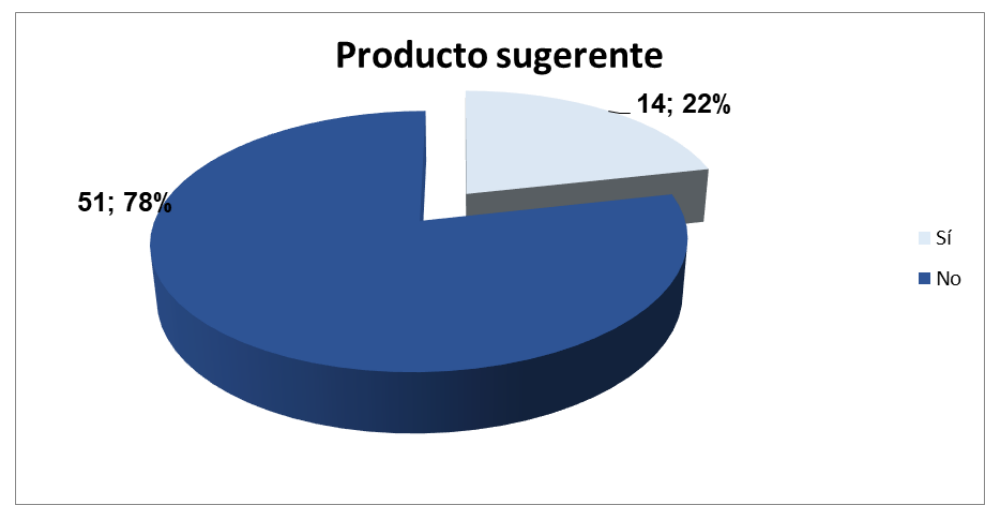

Figura 10. Producto sugerente. Fuente: Elaboración propia.

Ítem 7. Cliente repitente e ítem 8. Cliente Fiel

Según los resultados de las encuestas un 37\% ya había visitado la instalación anteriormente, y un 31\% siente fidelidad con la instalación al dirigirse a la misma siempre que necesita un producto de los que se ofrecen allí. Indicadores que son necesarios elevar debido a los beneficios que trae a un establecimiento los clientes de estas categorías (Figuras 11 y 12 ). 


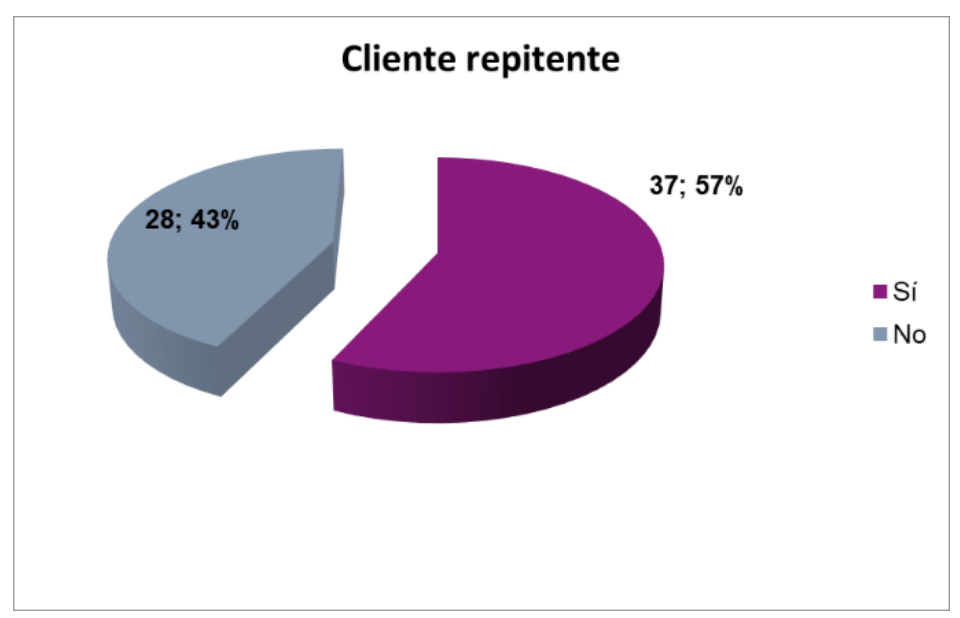

Figura 11. Cliente repitente. Fuente: Elaboración propia.

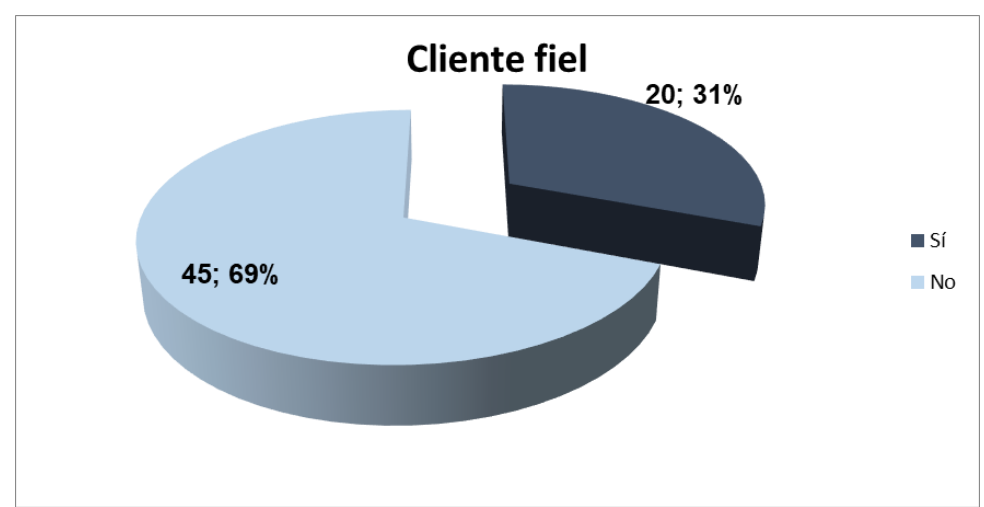

Figura 12. Cliente fiel. Fuente: Elaboración propia.

Ítem 9. Conocimiento de la marca

De los 65 encuestados solo 15 afirma tener conocimiento sobre la marca, para un 23\% respecto al total. A pesar de que la marca Giorgio es de gran prestigio a nivel internacional existen clientes que no la conocen, para ello resulta útil trabajar en la imagen de la tienda que tiene que estar en correspondencia con está y cumple además un papel importante la fuerza de venta que ha de ser capaz de inducir la compra exaltando las características del producto que ofertan (Figura 13). 


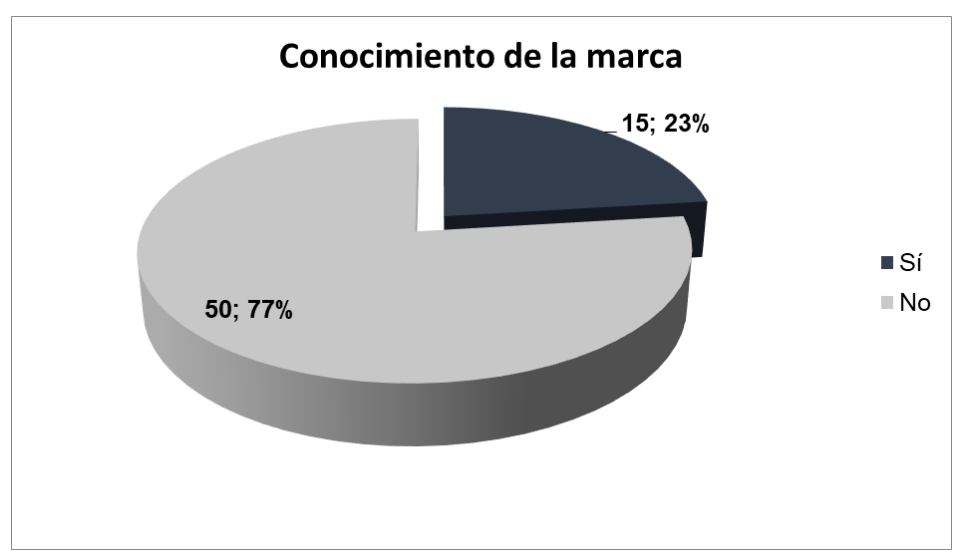

Figura 13. Conocimiento de la marca. Fuente: Elaboración propia.

Ítem 10. Relación calidad-precio

De los 65 encuestados, 28 la califican de muy buena, 30 de buena para un $89 \%$ de evaluaciones positivas, ello indica que un $21 \%$ lo califican negativamente. Sin embargo, la calidad de la marca Giorgio es reconocida. Ello sugiere la posibilidad de estudiar el precio de los mismos, o bien puede indicar una incompatibilidad de segmentos de mercados; pues esta tienda está dirigida a personas con un nivel adquisitivo superior a la media y su ubicación geográfica no la beneficia en todo sentido como a tiendas de la competencia ubicadas en el centro de la ciudad, donde fluyen más clientes con estas características. De lo anterior se debe la necesidad de una publicidad eficiente del producto (Figura 14).

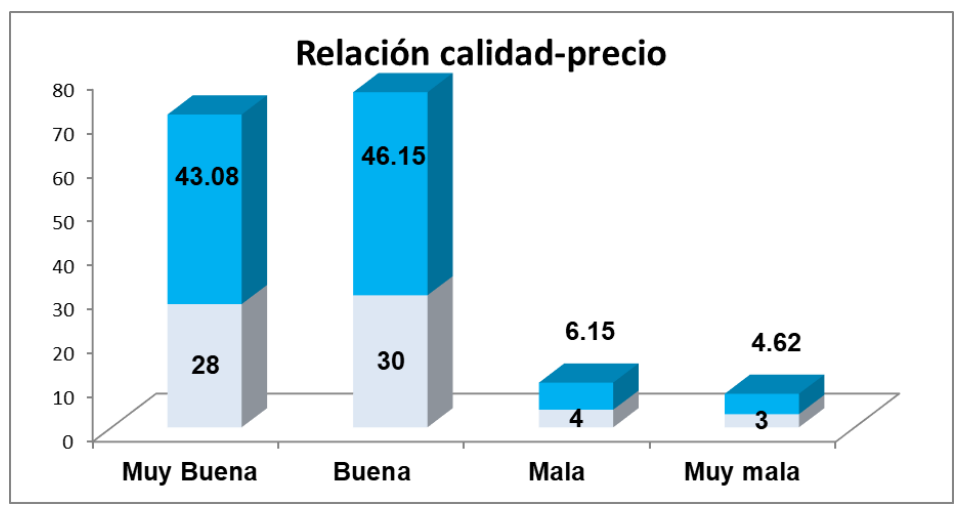

Figura 14. Relación calidad-precio. Fuente: Elaboración propia.

\section{ANÁLISIS DE LAS CAUSAS DE LAS DEFICIENCIAS ENCONTRADAS}

A partir del análisis de los resultados de las encuestas y del diagnóstico de la entidad se pudieron detectar varias problemáticas, cuyas causas fueron analizadas a partir de la construcción de un diagrama de causa efecto (Figura 15). 


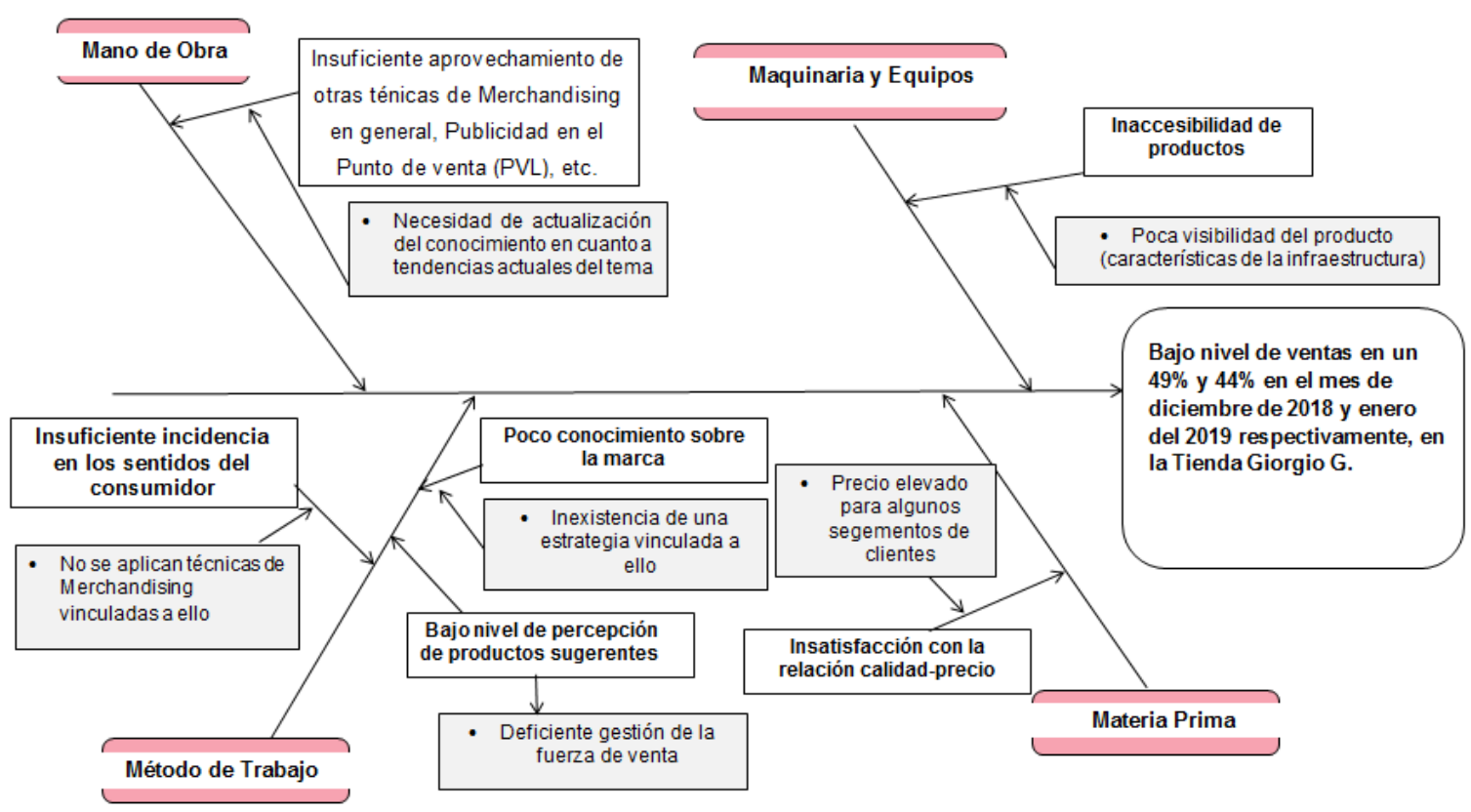

Figura 15. Diagrama de causa efecto

$\checkmark$ Inaccesibilidad de productos: está generada por una colocación ineficiente del producto que afecta la visibilidad del mismo.

$\checkmark$ Bajo nivel de percepción de productos sugerentes: está dado por una gestión ineficiente de la fuerza de venta al no tener identificado un producto a vender por sus características identificativas de la marca o porque su concepción genera esta necesidad.

$\checkmark$ Poco conocimiento sobre la marca que comercializa: generado por la inexistencia de una estrategia que busque elevar el conocimiento de la misma en el mercado.

$\checkmark$ Insatisfacciones con la relación calidad-precio: está dada principalmente por el precio del producto vinculado a una incompatibilidad de los segmentos de clientes que visitan la instalación.

$\checkmark$ Insuficiente incidencia en los sentidos del consumidor: no se aplican técnicas de Merchandising vinculada a ello.

$\checkmark$ Insuficiente aprovechamiento de otras técnicas de Merchandising, Publicidad en el Punto de venta (PVL) etc.: necesidad de actualización del conocimiento en cuanto a tendencias actuales del tema. 


\section{ACCIONES PARA CORREGIR LAS DEFICIENCIAS ENCONTRADAS}

Para dar solución a las deficiencias encontradas se elaboró un plan compuesto por 16 acciones en total a ejecutar.

Tabla 2.

Plan de acciones

\begin{tabular}{|c|c|c|c|c|}
\hline$\#$ & Problema & Causa & \# & Acción \\
\hline \multirow{3}{*}{1} & \multirow{3}{*}{$\begin{array}{l}\text { Inaccesibilidad de } \\
\text { productos }\end{array}$} & \multirow{3}{*}{$\begin{array}{l}\text { Colocación } \\
\text { ineficiente del } \\
\text { producto }\end{array}$} & 1 & $\begin{array}{c}\text { Realizar un análisis del comportamiento } \\
\text { de venta de cada producto según } \\
\text { inventario }\end{array}$ \\
\hline & & & 2 & $\begin{array}{c}\text { Colocar los productos estableciendo las } \\
\text { zona caliente y zona fría, según el } \\
\text { análisis del comportamiento de venta } \\
\text { de los mismos }\end{array}$ \\
\hline & & & 3 & $\begin{array}{c}\text { Exponer rotativamente en áreas visibles } \\
\text { los productos ubicados en el nivel de } \\
\text { sombrero }\end{array}$ \\
\hline \multirow{3}{*}{2} & \multirow{3}{*}{$\begin{array}{l}\text { Bajo nivel de } \\
\text { percepción de } \\
\text { productos } \\
\text { sugerentes }\end{array}$} & \multirow{3}{*}{$\begin{array}{l}\text { Gestión } \\
\text { ineficiente de la } \\
\text { fuerza de venta }\end{array}$} & 4 & $\begin{array}{l}\text { Identificar qué producto necesita de un } \\
\text { valor adicional para ser vendido por sus } \\
\text { características (a partir de la acción 1) }\end{array}$ \\
\hline & & & 5 & $\begin{array}{l}\text { Utilizar técnicas de presentación para } \\
\text { venderlos, entre ellas: sugerencia de } \\
\text { combinaciones con productos más } \\
\text { vendibles a partir de su exposición en el } \\
\text { lineal }\end{array}$ \\
\hline & & & 6 & $\begin{array}{l}\text { Sugerencia de combinaciones con } \\
\text { productos más vendibles a partir de la } \\
\text { publicidad en el punto de venta } \\
\text { propiciada por la fuerza de venta }\end{array}$ \\
\hline \multirow{2}{*}{3} & \multirow{2}{*}{$\begin{array}{c}\text { Poco } \\
\text { conocimiento } \\
\text { sobre la marca } \\
\text { que comercializa }\end{array}$} & \multirow{2}{*}{$\begin{array}{l}\text { Inexistencia de } \\
\text { una estrategia de } \\
\text { publicidad de la } \\
\text { marca }\end{array}$} & 7 & $\begin{array}{l}\text { Diseñar una estrategia de publicidad de } \\
\text { la marca en el punto de venta }\end{array}$ \\
\hline & & & 8 & $\begin{array}{l}\text { Gestionar el diseño e impresión de } \\
\text { sueltos y afiches con información sobre }\end{array}$ \\
\hline
\end{tabular}




\begin{tabular}{|c|c|c|c|c|}
\hline & & & & la marca \\
\hline & & & 9 & $\begin{array}{l}\text { Distribuir sueltos con información } \\
\text { sobre la marca Giorgio }\end{array}$ \\
\hline & & & 10 & $\begin{array}{l}\text { Colocar afiches con breve información } \\
\text { sobre la marca en concordancia con la } \\
\text { decoración del establecimiento }\end{array}$ \\
\hline \multirow{3}{*}{4} & \multirow{3}{*}{$\begin{array}{l}\text { Insatisfacciones } \\
\text { con la relación } \\
\text { calidad-precio }\end{array}$} & $\begin{array}{l}\text { Incompatibilidad, } \\
\text { precio-segmentos } \\
\text { de clientes }\end{array}$ & 11 & $\begin{array}{l}\text { Realizar un estudio del perfil del cliente } \\
\text { que compra en la entidad }\end{array}$ \\
\hline & & \multirow{2}{*}{$\begin{array}{l}\text { (Ubicación } \\
\text { geográfica) }\end{array}$} & 12 & $\begin{array}{l}\text { Gestionar la realización de modelajes, } \\
\text { entrega de sueltos, etc. en los lugares } \\
\text { que frecuenten estos clientes }\end{array}$ \\
\hline & & & 13 & $\begin{array}{l}\text { Crear una página web para } \\
\text { promocionar los productos, la marca } \\
\text { etc. }\end{array}$ \\
\hline \multirow[t]{2}{*}{5} & \multirow{2}{*}{$\begin{array}{l}\text { Insuficiente } \\
\text { incidencia en los } \\
\text { sentidos del } \\
\text { consumidor }\end{array}$} & \multirow{2}{*}{$\begin{array}{l}\text { Inaplicabilidad de } \\
\text { técnicas de } \\
\text { Merchandising } \\
\text { que estimulen los } \\
\text { sentidos }\end{array}$} & 14 & $\begin{array}{c}\text { Gestionar los medios técnicos } \\
\text { necesarios para poner música en la } \\
\text { entidad acorde a las características de } \\
\text { la misma }\end{array}$ \\
\hline & & & 15 & $\begin{array}{c}\text { Ambientar la instalación con un aroma } \\
\text { que los identifique }\end{array}$ \\
\hline 6 & $\begin{array}{c}\text { Insuficiente } \\
\text { aprovechamiento } \\
\text { de otras técnicas } \\
\text { de } \\
\text { Merchandising, } \\
\text { Publicidad en el } \\
\text { Punto de venta } \\
\text { (PVL) etc. }\end{array}$ & $\begin{array}{l}\text { Necesidad de } \\
\text { actualización del } \\
\text { conocimiento en } \\
\text { cuanto a } \\
\text { tendencias } \\
\text { actuales del tema }\end{array}$ & 16 & $\begin{array}{c}\text { Gestionar con el Centro de } \\
\text { Capacitación del Turismo la } \\
\text { capacitación de los trabajadores de la } \\
\text { entidad }\end{array}$ \\
\hline
\end{tabular}

Fuente: Elaboración propia 


\section{CONCLUSIONES}

Los indicadores de desempeño empresarial de la tienda Georgio $G$ muestran que las principales insuficiencias vinculadas al cumplimiento de las ventas se vinculan fundamentalmente a insuficiencias relacionadas con la materia prima y los métodos de trabajo empleados en la entidad. Este diagnóstico permitió definir 16 acciones encaminadas a mejorar estos problemas en la instalación, los cuales se encuentran en la actualidad en fase de implementación.

\section{REFERENCIAS BIBLIOGRÁFICAS}

Baek, E., Choo, H. J., \& Lee, S. H. (2018). Using warmth as the visual design of a store: Intimacy, relational needs, and approach intentions. Journal of Business Research, 88, 91-101. doi: https://doi.org/10.1016/j.jbusres.2018.03.013

Cassanego, J. P., María, F. T., \& Caren, R. (2011). Satisfacción y valoración del consumidor en relación al turismo de compras en la ciudad de Rivera, Uruguay Estudios y Perspectivas en Turismo, 20, 34-56.

Churchill, G., \& Peter, J. (2000). Marketing: criando valor para o cliente. Editora Saraiva.

Dholakia, R. R., Dholakia, N., \& Chattopadhyay, A. (2018). Indigenous marketing practices and theories in emerging economies: Consumer behavior and retail transformations in India. Journal of Business Research, 86, 406-415. doi: https://doi.org/10.1016/j.jbusres.2017.09.030

Kakatkar, C., \& Spann, M. (2018). Marketing analytics using anonymized and fragmented tracking data. International Journal of Research in Marketing. doi: https://doi.org/10.1016/i.ijresmar.2018.10.001

Kotler, P., \& Kevin, L. (2006). Dirección de Marketing. Recuperado de https://publiclina.files.wordpress.com/2015/01/direccion de marketing kotler edi12.pdf

Leyva del Toro, C. (2018) Metodología para la gestión de tiendas extrahoteleras. Retos turísticos, 16(2): 23-34.

Moutinho, L. (2000). Strategic Management in Tourism. Recuperado de http://citeseerx.ist.psu.edu/viewdoc/download?doi=10.1.1.1000.6137\&rep=rep1\&type=p df\#page $=14$ 
Perelló Cabrera, JL. 2005. Introducción a la Investigación de Mercados Turísticos. La Habana. Centro de Estudios Turísticos. UH.

Richers, R. (1984). O enigmático maisindispensável consumidor: teoria e pratica. Revista de Administração, 19, 45-56.

Rodrigues, F. F., \& Delane, B. (2012). The effect of factors related to Merchandising on retail sales. FORUM, 52.

Schiffman, L. (2010). Consumer Behavior. Recuperado de https://www.academia.edu/6116556/Comportamiento del Consumidor Schiffman 10a $\underline{E d}$

Serrano, L. B., Escalona, R. R., \& Pompa, D. F. (2018). Estudio bibliométrico de los artículos originales encontrados en sciencedirect sobre comportamiento del consumidor en tiendas. 